appearance of the enamel in various degrees of hypoplasia, and of the microscopic picture of the dentine : any uncalcified areas in the dentine are considered to be abnormal, but are so common that it is possible some authorities would consider them normal. Close agreement between the macroscopic and microscopic appearances was obtained in more than 85 per cent. of the examinations. Of the deciduous teeth, 14 per cent. were found to be perfectly calcified, 21 per cent. were slightly, and 64 per cent. definitely, hypoplastic. The incisors were the best calcified, 49 per cent. being normal : 8 per cent. of the canines, 7 per cent. of the first molars, and only 1 per cent. of the second molars could be so classed. Slighter defects appeared among the remaining incisors and canines, and the more severe degrees of hypoplasia were observed in the majority of the defective molars. The teeth obtained from private sources, as opposed to those from dental clinics, were less defective in structure. Of the permanent teeth examined, none was normal, and 92 per cent. showed definite hypoplasia; but these figures give no indication of the condition of permanent teeth in Great Britain, since all those examined had been extracted for caries, or to help in the adjustment of irregularities.

These results also indicate that there is less chance of interference with calcification before birth than afterwards, and that the more rapid the development of the tooth, the more defective is its structure likely to be. The defects in structure are probably to be correlated with the diet during the time the teeth are developing in the jaws: thus before birth, even if the mother is on a deficient diet, the foetus can obtain its requirements of salts and vitamins by the sacrifice of the maternal stores. After weaning, the child has to depend entirely on its food and on its own stores, which are likely to be low if the mother's diet has been poor, for its supply of the substances necessary for the proper development and calcification of the teeth. At the same time, the diet frequently contains a large amount of cereal products, the influence of which is exerted against proper calcification. The incisors are the most advanced in development at birth, and are also the best developed structurally. The second molars are the least developed at birth; they grow rapidly after birth, and have the worst structure of any of the teeth.

In conclusion, it may be said that diet affects the teeth as it affects the other tissues of the body, and that the teeth, like the other tissues, respond more easily to some defects in the diet than to others, but that the same defect rarely affects more than a few of the tissues to a marked degree. In addition, the diet can affect the teeth locally by causing alterations in their environment, the changes in the teeth then starting from their oral surfaces.

The degree to which diet directly affects the structure of the teeth depends in part on the definition of the terms 'normal' and 'abnormal' as regards this structure : and on this depends again the relationship between abnormality of structure and dental decay. In any case, caries will not be initiated unless acid is present on the oral surfaces of the tooth. It may be pointed out that it is difficult to produce caries in animals unless at the same time the conditions are such as to lead to defective structure also.

\title{
The Enhancement Principle in X-ray Photographs.
}

\section{By Sir William BragG, K.B.E., F.R.S.}

$\mathrm{A}^{\mathrm{N}}$ interesting phenomenon is often shown in the X-ray rotation photographs of crystals. Photographs of this kind are obtained by causing a crystal to revolve steadily about an axis perpendicular to the direction of a fine pencil of homogeneous X-rays. As the crystal revolves, one set of planes after another comes to a favourable condition for reflecting the pencil and a corresponding spot appears upon the plate. The crystal is usually very small indeed, and the shape of the crystal largely determines the form of the spot. The complete photograph shows an array of spots which displays certain regularities of arrangement as exemplified in Figs. 1, 2, and 3. When the photographic plate is flat, the spots arrange themselves on hyperbolæ as in Fig. 1 ; if a circular film is used the hyperbolæ are replaced by straight lines as in Figs. 2 and 3. The phenomenon to which attention is now directed consists in the enhancement, sometimes a very great enhancement, of certain groups of the spots. The explanation is more readily understood if consideration is first given to an analogous case of greater simplicity.

An ordinary optical grating gives a series of spectra : if the incident light is homogeneous, each spectrum is limited to a line. If now every fifth line in the grating were intensified or altered in some way, there would be added to the series already referred to a second series consisting of lines five times as close-packed, and every fifth line of the new spectrum would coincide with one of the old. For the sake of what follows, this may be put in another way.

If, in the first place, the grating had contained only lines corresponding to those which we have spoken of above as being intensified in some way, the more numerous series of spectra also referred to above would have appeared upon the plate. If, now, four other lines were intercalated uniformly between the lines already drawn upon the grating, every fifth line of the series of spectra would be enhanced.

From this simple case we may now proceed to the more complicated three-dimensional case of the crystal. A first example may be taken from the work on the structure of the silicates which has been carried out by Prof. W. L. Bragg and his colleagues at Manchester. They have shown that a silicate may be regarded in the first instance as a compilation of close-packed spherical oxygen atoms : the other atoms belonging to the silicate are to be thought of as inserted in the interstices in the

No. 3044, VoL. 121] 
close-packed structure. Sometimes the inserted atoms, for example, when they are beryllium or silicon, do not strain the structure, and again larger atoms such as calcium or magnesium distort it more or less. But in all cases the silicate structure may be described as consisting of a basis of close-packed oxygens on which a larger pattern of other atoms has been superimposed. This is the very condition which was exemplified in simpler form in the optical case already described. When the rotation photographs are considered, the parallel consequences are also to be found. Certain spots are strongly enhanced; these are the spots which would be given by the oxygens acting alone in close-packed structure. The multiplicity of other spots which fill the photograph, including those which have been enhanced, are such as would appear if the structure consisted only of all the atoms except the oxygens. An example of these photographs was given in Nature, Sept. 17, 1927, and is reproduced here (Fig. 1). This one picture is sufficient to show the exceedingly interesting and beautiful structure of the silicate family.

A second example may be taken from the work of

two accompanying illustrations (Figs. 2 and 3 ) that whole rows of spots are here enhanced. Proceeding upwards or downwards from the central horizontal

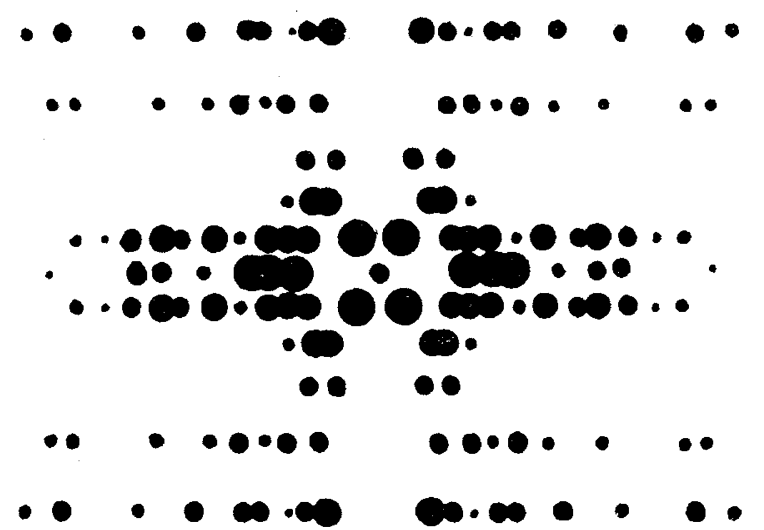

Fic. 2.-Rotation photograph about the $c$-axis of anthracene. The size of a spot is intended to represent its strength on the photographic film.

row, which is called the equator, the first row in each case is still strong; but in naphthalene the second row almost fails, and in anthracene the second and third. There is, in fact, a maximum between the third and fourth rows in naphthalene and the fourth and fifth rows in anthracene. The succeeding minima of intensity of rows are not shown in these pictures, because it is somewhat inconvenient to obtain photographs sufficiently extended upwards and downwards to include them, but it is easy to find

FIG. 1.-Rotation photograph around $b$-axis of disthene (right) compared with ideal rotation photograph around cube edge for close-packed atoms (left). Spots in the right-hand diagram corresponding to spots in the left-hand diagram are contained in circles.

Dr. Müller on the long chain compounds (Proc. Roy. Soc., vol. 114, p. 542). In this case also there are two periodicities in the structure. There is that which depends on the length of the chain, which in stearic acid is $48.84 \mathrm{~A}$. Along the axis of the chain there is a second and finer periodicity due to the regular arrangement of the carbon atoms. The repetition takes place every second atom and its length is 2.52: the one periodicity is approximately nineteen times that of the other. A rotation photograph of stearic acid shows a multiplicity of spots due to the many sets of planes which are capable of reflecting the homogeneous X-rays usually employed. Some of these spots are very strongly enhanced for similar reasons to those already given. For example, the sets of planes denoted by $(2,0,19),(2,0,38),(0,1,20),(0,2,19)$, $(0,3,19),(0,0,18),(0,0,20)$, all show up on the plate, and indeed are almost the only high indices planes to appear. Such sets divide the $c$-axis into 18, $19,20 \ldots$ equal parts, as their designation implies. Consequently, their periodicities nearly coincide with that of the carbons of the chain. It is not necessary that the one periodicity should be an exact submultiple of the other.

A third example may be taken from the photographs of naphthalene and anthracene about the $c$-axis. It will be seen from a comparison of the out from other observations, which need not be given here, that minima do follow and are succeeded again by maxima. Thus, for example, the eighth and ninth rows of anthracene are strong. This enhancement of whole rows means

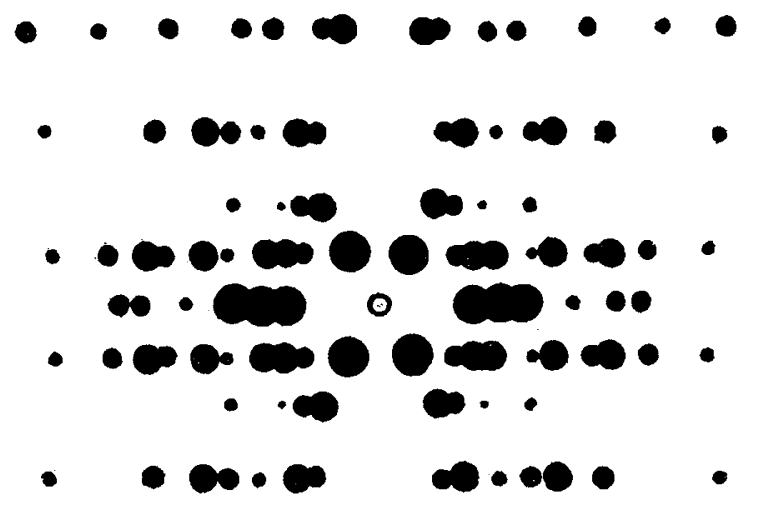

FIG. 3.-Rotation photograph about the $c$-axis of naphthalene.

that there is a certain repetition along the $c$-axis of a distribution of matter which in the case of naphthalene divides it into between three and four

No 3044, VoL. 121] 
parts ; in the case of anthracene, between four and five. This is just what is to be expected if the structure of these crystals is that which was given by the author some years ago. The axis of a crystal in each case lies along the $c$-axis and the repetition is of the width of a benzene ring, namely, $2 \cdot 52$. The length of the $c$-axis of naphthalene is 8.69 and of anthracene $11 \cdot 18$. The axis of the former case is $3 \frac{1}{2}$ times the width of the ring, and of the latter case about $4 \frac{1}{2}$ times. The enhancement of the ninth row is due to the fact that the diameter of the ring divides it into two equal halves; there are periodicities of both $\mathbf{1 . 2 6}$ and 2.52 along the $c$-axis. The distribution of the intensities of the various lines in these figures is, in fact, an index of the distribution of matter or electrons along the axis about which the crystal was rotated.

There is one further point of interest in these last photographs. It will be observed that the distribution of spots on the equator is exactly the same for the two crystals. Even in the next row there is very little difference for the two, though the difference increases with the distance of the rows from the equator. Assuming the structure of the crystals to be that already referred to, to an eye looking along the $c$-axis the crystals would seem exactly alike. To what may be called to an X-ray eye looking in the same direction the crystals would look very nearly alike, but there would be a small difference because such an eye would see in depth as well as in plan. The eye would not observe, and the $\mathrm{X}$-ray eye would, that certain atoms were hiding behind one another. The spots on the equator are due to planes which pass through the $c$-axis. The very close correspondence between the positions and intensities of the equator spots in the two crystals shows that to an eye, supposed capable of looking along the $c$-axis and observing the disposition of the atoms, the two crystals would appear exactly the same. This is confirmatory evidence of the structures assigned to them.

A somewhat different use of the same principle has been made by W. T. Astbury in his work on the acetyl-acetones (Proc. Roy. Soc., 112, p. 457). From a consideration of the relative intensities of the rows, he has drawn conclusions as to the relative positions of the molecules in the unit cell of the crystal.

\section{Obituary.}

Mr. AleXander Stemens.

LEXANDER SIEMENS was born in Hanover $A$ in January 1847. He belonged to the second generation of the four brothers Siemens whose names are so well known. He used to tell how his parents owed allegiance to the King of England until 1837, when, under the Salic Law, Hanover was separated from England and given to the Duke of Cumberland, the fifth son of George III. In 1866 Hanover was annexed by Prussia, and Siemens automatically became a Prussian. $\mathrm{He}$ was educated at Hanover and Berlin, and in $\mathbf{1 8 6 7}$ entered the telegraph workshops of Siemens' Brothers at Woolwich, of which Sir William Siemens, the first president of the Institution of Electrical Engineers, was a director. $\mathrm{He}$ was then employed in the erection of the Indo-European telegraph line in Persia and in laying cables in the Black Sea. He also worked in the cable ship Faraday. He served in the German Army during the Franco-Prussian War and was awarded the Iron Cross. In 1878 he became a naturalised British subject.

After his release from the German Army, Siemens came to England and assisted Sir William Siemens in developing the regenerative furnace. About 1879 he developed a system of lighting public halls by means of arc lamps. The systems adopted at the Albert Hall and in the British Museum Reading Room were done under his direction. He also carried out the electric lighting of Godalming, the first town in England to have an electric light supply.

Siemens had the greatest faith in science, in scientific training, and especially in scientific management. He considered that scientific management was quite as important as either capital or labour in developing the industry of the country. He was enthusiastically in favour of the decimal system, and in 1902 strongly advocated its universal adoption in a discussion held at the Institution of Electrical Engineers. His great protagonist was the late Sir Frederick Bramwell. The sympathies of most of the auditors were in favour of the decimal system, but the older generation of engineers seemed to think that engineering in Great Britain would be hopelessly handicapped internationally were we to abolish the inch and the pound. There was one thing said at this meeting which the writer never saw contradicted, and that was that without the decimal system it would not be possible to extract square roots. It is quite easy, however, to turn the square root of any number or fraction into a continued fraction and then find its value to any required degree of accuracy as a vulgar fraction.

Siemens was president of the Institution of Electrical Engineers in 1894 and 1904, and of the Institution of Civil Engineers in 1910. In his presidential address in 1894 he discussed, among other subjects, the possibility of trebling the speed of our trains. Although he considered it rash to say that such a speed could not be obtained, yet the necessity of strengthening the permanent way, etc., made it very unlikely that it would be adopted commercially. $\mathrm{He}$ contributed many papers and gave many lectures to scientific bodies. One of his. most interesting papers was an account of experiments carried out by German engineers on the military railway connecting Marienfelde and Zossen. In these experiments, train speeds up to 125 miles per hour were obtained. The A.E.G. Co. insured the lives of their engineers before the experiments. After the tests, the front of the engine was coated with dead insects, and amongst them a dead swallow. The speeds 